\title{
Arañas (Arachnida: Araneae) asociadas a dos bosques degradados del Chaco húmedo en Corrientes, Argentina
}

\author{
Gilberto Avalos ${ }^{1}$, Gonzalo D. Rubio ${ }^{1}$, María E. Bar ${ }^{1}$ \& Alda González ${ }^{2}$ \\ 1 Cátedra de Artrópodos, Facultad de Ciencias Exactas y Naturales, Universidad Nacional del Nordeste, Avda. Libertad \\ 5470 (3400) Corrientes, Argentina; gilbertoa@exa.unne.edu.ar \\ 2 Centro de Estudios Parasitológicos y de Vectores (CEPAVE)(UNLP), Calle 2 N$^{\circ} 584$ (1900), La Plata, Argentina; \\ asgonzalez@cepave.edu.ar
}

Recibido 24-V-2006. Corregido 08-XII-2006. Aceptado 07-V-2007.

\begin{abstract}
Spiders (Arachnida: Araneae) associated with two degraded forests in the humid Chaco of Corrientes, Argentina. The advancing degradation of the forest in the biogeographic Chaco province (Argentina) produces an important loss of its little known biodiversity. We studied the spider biodiversity in two forests of Corrientes, Argentina's "Distrito Oriental Húmedo del Chaco": Laguna Brava and El Perichón. Seasonal samplings of foliage and fallen leaves between 2001 and 2002 produced 2067 individuals from 33 families and 226 species/ morphospecies). The families Araneidae, Anyphaenidae, Salticidae and Theridiidae were the most abundant in both forests. The "orb weavers" guild had the highest number of specimens $(\mathrm{n}=382)$ and "stalkers" the highest richness $(\mathrm{S}=56)$. In Brava, highest abundance was in the summer $(\mathrm{n}=287)$ and spring $(\mathrm{n}=273)$, in Perichón, it was in winter $(\mathrm{n}=315)$. The specific richness and the diversity indexes were higher in Brava $\left(\mathrm{S}=134, \mathrm{H}^{\prime}=4.23, \mathrm{E}=0.86, \mathrm{D}=0.023\right)$ than in Perichón $\left(\mathrm{S}=127, \mathrm{H}^{\prime}=4.08, \mathrm{E}=0.84, \mathrm{D}=0.029\right)$. The similarity value between both forests was MH=0.611. Rev. Biol. Trop. 55 (3-4): 899-909. Epub 2007 December, 28.
\end{abstract}

Key words: Araneae, biodiversity, humid forests, Corrientes, Argentina.

Las arañas comprenden un grupo faunístico diverso y ampliamente distribuido en todos los ecosistemas terrestres, incluso el dulceacuícola (Turnbull 1973). Su diversidad es particularmente elevada en los bosques tropicales, donde se puede encontrar casi el $80 \%$ de las especies conocidas (Coddington y Levi 1991). Son depredadores generalistas importantes en las redes tróficas por su abundancia, biomasa y diversidad de especies. Por sus hábitos depredadores, influyen en la densidad y actividad de la fauna de detritívoros y fungívoros, afectando los procesos de descomposición (Wise 2002).

Las arañas han ganado una amplia aceptación en los estudios ecológicos como indicadores de calidad ambiental (Clausen 1986, Maelfait et al. 1990, Willett, 2001, PinkusRendón et al. 2006, Tsai et al. 2006), ya que las comunidades de arañas han mostrado ser fuertemente influenciadas, y de manera predecible, por el tipo de hábitat y el patrón de uso de la tierra (Weeks y Holtzer 2000). La arquitectura de la vegetación juega un papel importante en la composición de especies encontradas dentro de un hábitat (Scheidler 1990), por lo que una vegetación estructuralmente compleja puede contener una abundancia y diversidad mayor de arañas (Hatley y MacMahon 1980).

A pesar de ser consideradas uno de los grupos entomófagos más abundantes en la naturaleza (Nyffeler et al. 1994) y del esfuerzo de algunos autores (Höfer 1990, Silva 1996, Silva y Coddington 1996), que en los últimos años contribuyeron al conocimiento de las comunidades de arañas en bosques del Neotrópico, el conocimiento que de ella se tiene es aún incipiente. 
En Argentina el conocimiento taxonómico y sistemático de algunas familias de arañas es amplio. Sin embargo, las investigaciones referidas a aspectos ecológicos de comunidades de arañas asociadas a áreas naturales o alteradas son escasas. Se destacan los estudios efectuados en un área natural protegida de la provincia de Formosa (Corronca y Abdala 1994).

Las actividades humanas han causado severos cambios en la composición y la diversidad de la mayoría de los ecosistemas conocidos (Barnes et al. 1998), llevando a la modificación de las poblaciones, la distribución de las especies, la estructura y el funcionamiento de las comunidades, pudiendo llegar aún a la extinción (Meffe y Carroll 1994). Por lo mencionado, este trabajo tiene como objetivos conocer la fauna de arañas en dos bosques degradados del chaco húmedo argentino de la provincia de Corrientes y analizar comparativamente la diversidad, riqueza específica y abundancia según las estaciones climáticas.

\section{MATERIALES Y MÉTODOS}

Área de estudio: el área estudiada pertenece al Distrito Oriental Húmedo de la Provincia Fitogeográfica Chaqueña (Cabrera y Willink 1980, Morrone 2001); con clima subtropical húmedo con veranos calurosos y lluviosos e inviernos fríos y secos, temperatura y humedad media de $21.3^{\circ} \mathrm{C}$ y $1200 \mathrm{~mm}$, respectivamente (Bruniard 1981). Los bosques se ubican en las localidades de Laguna Brava (2731' S, 58 $41^{\circ}$ ' W) y El Perichón (27²4' S, 58 $44^{\circ}$ 'W), departamento Capital, Corrientes, Argentina. Ambos bosques están degradados por tala de árboles de importancia económica y por pastoreo. El Perichón se encuentra sobre el Río Paraná y presenta una vegetación más cerrada y densa, con un microclima húmedo, fresco y con poca luz. Laguna Brava está ubicada en una loma entre zonas deprimidas ocupadas por bajos inundables con vegetación higrófila. Un matorral de abrigo muy denso dificulta la entrada, la luz es muy pobre y el microclima muy húmedo, con abundancia de epifitos (orquídeas, cactáceas y helechos), encontrándose pequeños charcos temporarios.

En los dos bosques el estrato de árboles se caracteriza por la presencia de quebracho colorado (Schinopsis balansae Engl.), urunday (Astronium balansae Engl.) y ejemplares de Tabebuia heptaphylla (Vell.) Toledo, Enterolobium contortisiliquum (Vell.) Morong y Patagonula americana L.; en el estrato arbustivo predominan Schaefferia argentinensis Speg., Phylira brasiliensis Klotzsch, Trichilia elegans A. Juss. y Celtis iguanaea (Jacq.) Sarg.; y en el estrato herbáceo se encuentran Oplismenus hirtellus (L.) P. Beauv., Pseudananas sagenarius (Arruda) Camargo, Anemia tomentosa (Savigny) Sw. y Adiantopsis chlorophylla (Sw.) Fée.

Trabajo de campo: los muestreos se efectuaron desde mayo 2001 hasta marzo de 2002, de acuerdo al siguiente esquema: otoño (mayo y junio), invierno (agosto y septiembre), primavera (noviembre y diciembre) y verano (febrero y marzo). Las arañas fueron recolectadas mediante la técnica de golpeteo del follaje en los estratos arbóreo y arbustivo y el tamizado de la hojarasca en el mantillo (Coddington et al. 1991). En cada bosque, se muestreó entre las 9:00-12:00 h, en un área de $90000 \mathrm{~m}^{2}$ elegida al azar donde se tomaron, también al azar, cuatro muestras con cada una de las técnicas de colecta mencionadas. Cada muestra de vegetación consistió en 15 golpes con una vara sobre la vegetación arbustiva y porción baja del estrato arbóreo y el material fue recolectado sobre una sábana blanca de $2.25 \mathrm{~m}^{2}$. Las arañas de hojarasca fueron obtenidas por tamizado de una superficie de $0.25 \mathrm{~m}^{2}$ por muestra, las que fueron colocadas en bolsas de polipropileno debidamente rotuladas y fijadas en alcohol al $70 \%$ hasta su traslado al laboratorio.

En el laboratorio el material fue separado en familias y se identificó a nivel de especies y/o morfoespecies (msp) mediante el uso de claves y en algunos casos con la colaboración de especialistas del país. Las arañas fueron separadas por estado del desarrollo, sexo y clasificado por gremios (Enders 1976, Dippenaar-Schoeman et al.1989, Marc y Canard 1997, Uetz et al. 
1999). El material recolectado se depositó en la colección de la Cátedra de Artrópodos de la Facultad de Ciencias Exactas y Naturales, Universidad Nacional del Nordeste (UNNE) (CARTROUNNE).

Análisis estadísticos: para el análisis de datos se utilizó el programa estadístico Bio-Dap (Gordon y Douglas 1988: Bio-Dap, Ecological Diversity y its Measurement), se calculó la riqueza de especies (S), la diversidad mediante los índices de Shannon-Wienner $\left(\mathrm{H}^{\prime}\right)$, de Simpson (D) y de equidad (E) y la similitud con el índice de Morisita-Horn (MH).

Para determinar si el ambiente ha sido suficientemente muestreado se utilizó el estimador CHAO1 (Colwell y Coddington 1994): $\mathrm{S} 1=$ Sobs $+\left(\mathrm{a}^{2} / 2 \mathrm{~b}\right)$, donde Sobs corresponde al número de especies/msp observadas en la localidad, a es el número de especies/msp con un solo individuo y b es el número de especies/ msp con dos individuos en la localidad.

\section{RESULTADOS}

El total de arañas recolectadas en los dos bosques fue 2067 individuos pertenecientes a 33 familias y 226 especies/msp. Los juveniles $(\mathrm{n}=1$ 526) fueron más numerosos que los adultos y las hembras representaron el $68.6 \%$ (371/541) de la población adulta.

En Laguna Brava se hallaron 1022 ejemplares pertenecientes a 26 familias y 134 especies/msp y en El Perichón 1 045, pertenecientes a 30 familias y 127 especies/msp, todos del Infraorden Araneomorphae (Cuadro 1). Tres familias (Dictynidae, Ctenidae y Scytodidae) se encontraron sólo en Laguna Brava y siete (Amaurobiidae, Deinopidae, Palpimanidae, Prodidomidae, Segestriidae, Theridiosomatidae y Zodariidae) sólo en El Perichón.

Las familias más abundantes fueron: Araneidae $(n=139)$, Linyphiidae $(n=129)$, Anyphaenidae $(\mathrm{n}=121)$, Salticidae $(\mathrm{n}=90)$ y Thomisidae $(n=85)$ en Laguna Brava; Anyphaenidae $(n=166)$, Araneidae $(n=161)$, Salticidae $(n=118)$, Theridiidae $(n=112)$ en El Perichón. El resto de las familias presentó una abundancia menor a $n=80$ en los dos bosques estudiados. Entre las familias de mayor riqueza específica se destacaron Araneidae $(\mathrm{S}=26)$, Salticidae $(\mathrm{S}=22)$, Theridiidae $(\mathrm{S}=12)$ y Linyphiidae $(\mathrm{S}=10)$ en Laguna Brava; y Salticidae $(\mathrm{S}=23)$, Araneidae $(\mathrm{S}=18)$ y Theridiidae $(\mathrm{S}=13)$ en El Perichón. El resto de las familias mostraron una riqueza específica menor a $\mathrm{S}=10$ en los dos bosques estudiados. En Laguna Brava se registraron 33 especies/ msp con un solo individuo (singletons) y en El Perichón 32. Las especies/msp con dos individuos (doubletons) fueron 18 en ambas localidades (Cuadro 1).

Al comparar los dos bosques surge que Laguna Brava, si bien presenta menor abundancia de arañas, posee mayor riqueza específica y mayor diversidad y equidad $\left(\mathrm{H}^{\prime}=4.23\right.$, $\mathrm{D}=0.023, \mathrm{E}=0.86)$, que El Perichón $\left(\mathrm{H}^{\prime}=4.08\right.$, $\mathrm{D}=0.029, \mathrm{E}=0.84)$. La similitud entre ambos bosques fue $\mathrm{MH}=0.611$.

Las familias recolectadas en los dos bosques se organizaron en 8 gremios (Cuadro 2). Considerando la suma de los dos bosques, las arañas constructoras de telas orbiculares totalizaron el mayor número de individuos $(\mathrm{n}=382)$ y el gremio de las arañas cazadoras al acecho presentó la mayor riqueza de especies $(\mathrm{S}=56)$. En Laguna Brava los gremios más abundantes correspondieron a constructoras de telas sábanas $(n=221)$, constructoras de telas orbiculares $(n=170)$, cazadoras por emboscadas $(n=149)$ y cazadoras al acecho $(\mathrm{n}=140)$, el resto de los gremios con un número menor a 130 individuos; y los gremios con mayor número de especies fueron constructoras de telas orbiculares y cazadoras al acecho $(S=30)$, seguida por las constructoras de telas espaciales $(S=19)$, cazadoras por emboscada ( $\mathrm{S}=16)$ y vagabundas del suelo $(\mathrm{S}=15)$, los restantes con menos de 15 especies. En El Perichón los gremios más abundantes correspondieron a: constructoras de telas orbiculares $(\mathrm{n}=212)$, vagabundas de vegetación $(\mathrm{n}=181)$, cazadoras al acecho $(\mathrm{n}=157)$ y constructoras de telas sábanas $(n=137)$, el resto de los gremios representados por menos de 130 individuos; y los gremios con mayor riqueza específica fueron: cazadoras al acecho $(\mathrm{S}=29)$, 
CUADRO 1

Familias y especies/morfoespecies de arañas asociadas a dos bosques del Chaco Húmedo

(Corrientes, Argentina) (2001- 2002)

TABLE 1

Families and species/morphospecies of spiders associated with two forests of the Humid Chaco (Corrientes, Argentina)(2001-2002)

Familia

Amaurobiidae

Anyphaenidae

Araneidae

Corinnidae

Ctenidae

Deinopidae

Dictynidae

Gnaphosidae

Hahniidae

Hersiliidae

Linyphiidae

Lycosidae

Mimetidae

Oonopidae

Oxyopidae

Palpimanidae

Philodromidae

Pholcidae

Pisauridae

Prodidomidae

Salticidae

Scytodidae

Segestriidae

Selenopidae

Senoculidae

Sparassidae

Tetragnathidae

Theridiidae

Theridiosomatidae

Thomisidae

Titanoecidae

Uloboridae

Zodariidae

Especie/morfoespecie

\section{Laguna Brava}

ninguna

Yessica sp.- Aysha sp.- Teudis sp.

Wulfila sp.- 3 morfoespecies

Acacesia sp.- Aculepeira sp.- Alpaida sp.

Araneus sp.- Argiope argentata

Cyclosa sp. - Eustala sp.- Mangora sp.

Mecynogea sp.- Metepeira sp.

Micrathena sp.- Neoscona sp.

Ocrepeira sp.- Parawixia sp.

Pronous sp.- 11 morfoespecies

Trachelas sp.- Trachelopachys sp.

Orthobula sp.- 3 morfoespecies

1 morfoespecie

ninguna

2 morfoespecies

1 morfoespecie

Austrohahnia praestans

Tama sp.

Microlinyphia sp.- 9 morfoespecies

Aglaoctenus lagotis- 2 morfoespecies

Gelanor altithorax-Gelanor sp.

Mimetus sp.- 1 morfoespecie

Gamasomorpha wasmanniae

Neoxyphinus ogloblini- Oonops sp.

Scaphiella sp.

Hamataliwa sp. 1- Oxyopes sp. 1

Oxyopes sp. 2- Peucetia sp.

ninguna

4 morfoespecies

Guaranita munda- 4 morfoespecies

2 morfoespecies

ninguna

22 morfoespecies

Scytodes sp.

ninguna

Selenops maranhensis- Selenops sp.

Senoculus sp.

Polybetes rapidus

Leucauge sp.- Tetragnatha sp.

Argyrodes sp.- Episinus sp.

Theridion sp.- 9 morfoespecies

Ninguna

9 morfoespecies

Goeldia sp.

Conifaber guarani- Uloborus trilineatus

Ninguna
El Perichón

3 morfoespecies

Yessica sp.- Aysha sp.- Teudis sp.-

Wulfila sp.- Anyphaena sp.- 3 morfoespecies

Aculepeira sp.- Alpaida sp.

Amazonepeira sp.- Araneus sp.

Mangora sp.- Metepeira sp.

Micrathena sp. -Ocrepeira sp.

Parawixia sp.- Pronous sp.

8 morfoespecies

Trachelas sp.- Orthobula sp.

4 morfoespecies

ninguna

Deinopis sp.

ninguna

2 morfoespecies

Austrohahnia praestans

Tama sp.

8 morfoespecies

Aglaoctenus lagotis- 3 morfoespecies

Mimetus penicillatus-Gelanor $\mathrm{sp}$.

1 morfoespecie

Neoxyphinus ogloblini- Oonops sp.

Scaphiella sp.

Hamataliwa sp. 2- Oxyopes sp. 2

Peucetia sp.

Otiothops inflautus

4 morfoespecies

2 morfoespecies

2 morfoespecies

1 morfoespecie

23 morfoespecies

ninguna

Ariadna boesembergi

Selenops maranhensis- Selenops sp.

Senoculus sp.

Polybetes trifoveatus - Polybetes rapidus

Polybetes trilineatus- Polybetes sp.

Leucauge sp.- Tetragnatha sp.

Argyrodes sp.- Episinus sp.

Steatoda sp.- 10 morfoespecies

Epeirotypus sp.

5 morfoespecies

Goeldia sp.

Conifaber guarani- Uloborus trilineatus

Cybaeodamus sp. 
CUADRO 2

Riqueza especifica y abundancia de familias de arañas asociadas a dos bosques del Chaco Húmedo (Corrientes, Argentina, 2001-2002), agrupadas por gremio

TABLE 2

Species richness and family abundance of spiders associated with two forests of the Humid Chaco (Corrientes, Argentina)(2001-2002), by guild

\begin{tabular}{|c|c|c|c|c|}
\hline \multirow{2}{*}{ Gremio } & \multirow{2}{*}{ Familia } & \multicolumn{2}{|c|}{ Especie/morfoespecie } & \multirow{2}{*}{ Total } \\
\hline & & Laguna Brava & El Perichón & \\
\hline \multirow[t]{6}{*}{ СТO } & Araneidae & $26(139)$ & $18(161)$ & $35(300)$ \\
\hline & Tetragnathidae & $2(19)$ & $2(34)$ & $2(53)$ \\
\hline & Uloboridae & $2(12)$ & $2(13)$ & $2(25)$ \\
\hline & Deinopidae & $0(0)$ & $1(2)$ & $1(2)$ \\
\hline & Theridiosomatidae & $0(0)$ & $1(2)$ & $1(2)$ \\
\hline & Subtotal & $30(170)$ & $24(212)$ & $41(382)$ \\
\hline \multirow[t]{5}{*}{ CTS } & Linyphiidae & $10(129)$ & $8(47)$ & $18(176)$ \\
\hline & Amaurobiidae & $0(0)$ & $3(46)$ & $3(46)$ \\
\hline & Titanoecidae & $1(66)$ & $1(9)$ & $1(75)$ \\
\hline & Hahniidae & $1(15)$ & $1(35)$ & $1(50)$ \\
\hline & Subtotal & $12(210)$ & $13(137)$ & $23(347)$ \\
\hline \multirow[t]{4}{*}{ VV } & Anyphaenidae & $7(121)$ & $8(166)$ & $11(287)$ \\
\hline & Sparassidae & $1(1)$ & $4(6)$ & $4(7)$ \\
\hline & Selenopidae & $2(4)$ & $2(9)$ & $2(13)$ \\
\hline & Subtotal & $10(126)$ & $14(181)$ & $17(307)$ \\
\hline \multirow[t]{10}{*}{ VS } & Lycosidae & $2(9)$ & $3(35)$ & $5(44)$ \\
\hline & Gnaphosidae & $1(36)$ & $2(26)$ & $3(62)$ \\
\hline & Ctenidae & $1(3)$ & $0(0)$ & $1(3)$ \\
\hline & Corinnidae & $6(27)$ & $6(24)$ & $10(51)$ \\
\hline & Oonopidae & $4(19)$ & $3(16)$ & $4(35)$ \\
\hline & Zodariidae & $0(0)$ & $1(7)$ & $1(7)$ \\
\hline & Palpimanidae & $0(0)$ & $1(3)$ & $1(3)$ \\
\hline & Scytodidae & $1(16)$ & $0(0)$ & $1(16)$ \\
\hline & Prodidomidae & $0(0)$ & $1(3)$ & $1(3)$ \\
\hline & Subtotal & $15(110)$ & 17 (114) & 27 (224) \\
\hline \multirow[t]{4}{*}{$\mathrm{CA}$} & Salticidae & $22(90)$ & $23(118)$ & 45 (208) \\
\hline & Mimetidae & $4(16)$ & $3(24)$ & $6(40)$ \\
\hline & Oxyopidae & $4(34)$ & $3(15)$ & $5(49)$ \\
\hline & Subtotal & $30(140)$ & $29(157)$ & $56(297)$ \\
\hline
\end{tabular}


CUADRO 2 (Continuación)

Riqueza especifica y abundancia de familias de arañas asociadas a dos bosques del Chaco Húmedo (Corrientes, Argentina, 2001-2002), agrupadas por gremio

TABLE 2 (Continued)

Species richness and family abundance of spiders associated with two forests of the Humid Chaco (Corrientes, Argentina)(2001-2002), by guild

\begin{tabular}{llccc}
\multirow{2}{*}{ Gremio } & Familia & \multicolumn{2}{c}{ Especie/morfoespecie } & Total \\
CE & Laguna Brava & El Perichón & $14(149)$ \\
& Thomisidae & $9(85)$ & $5(64)$ & $8(56)$ \\
& Philodromidae & $4(36)$ & $4(20)$ & $4(31)$ \\
& Pisauridae & $2(17)$ & $2(14)$ & $1(20)$ \\
& Hersiliidae & $1(11)$ & $1(9)$ & $27(256)$ \\
& Subtotal & $16(149)$ & $12(107)$ & $23(185)$ \\
CTE & Therididae & $12(73)$ & $13(112)$ & $7(47)$ \\
& Pholcidae & $5(36)$ & $2(11)$ & $2(3)$ \\
& Dictynidae & $2(3)$ & $0(0)$ & $32(235)$ \\
& Subtotal & $19(112)$ & $15(123)$ & $1(3)$ \\
CTT & Segestriidae & $0(0)$ & $1(3)$ & $1(10)$ \\
& Lycosidae $($ A. lagotis $)$ & $1(4)$ & $1(2)$ & $3(19)$ \\
& Senoculidae & $1(1)$ & $1(9)$ & $226(2067)$ \\
& Subtotal & $2(5)$ & $3(14)$ &
\end{tabular}

$\mathrm{CTO}=$ constructoras de telas orbiculares, $\mathrm{CTS}=$ constructoras de telas sábanas, $\mathrm{VV}=$ vagabundas de vegetación, $\mathrm{VS}=$ vagabundas del suelo, $\mathrm{CA}=$ cazadoras al acecho, $\mathrm{CE}=$ cazadoras por emboscada, $\mathrm{CTE}=$ constructoras de telas espaciales, $\mathrm{CTT}=$ constructoras de telas tubulares.

$\mathrm{CTO}=$ orb weavers, $\mathrm{CTS}=$ sheet web-builders, $\mathrm{VV}=$ foliage runners, $\mathrm{VS}=$ ground runners, $\mathrm{CA}=$ stalkers, $\mathrm{CE}=$ hunting ambushers, $\mathrm{CTE}=$ space web-builders, $\mathrm{CTT}=$ tangle weavers.

constructoras de telas orbiculares $(S=24)$, vagabundas del suelo $(S=17)$ y constructoras de telas espaciales $(\mathrm{S}=15)$, los restantes gremios con menos de 15 especies.

El análisis por estación climática permitió evidenciar variaciones a lo largo del año. En Laguna Brava la mayor cantidad de individuos se observó en verano $(\mathrm{n}=287,28.1 \%)$ y en primavera $(\mathrm{n}=273,26.7 \%)$, correspondiéndole también a este último mes los mayores valores de riqueza específica $(\mathrm{S}=73)$, diversidad $\left(\mathrm{H}^{\prime}=3.82\right)$ y equitatividad $(\mathrm{E}=0.89)$. En El Perichón la mayor abundancia se constató en invierno $(n=315,30.1 \%)$ y en otoño $(n=262$,
$25.1 \%$ ) y la mayor cantidad de especies en invierno $(\mathrm{S}=71)$, no obstante la diversidad $\left(\mathrm{H}^{\prime}=3.72\right)$ y equitatividad $(\mathrm{E}=0.9)$ fueron superiores en verano. Asimismo, la mayor dominancia de especies $(\mathrm{D}=0.05)$ coincidió en invierno en ambos bosques (Fig. 1). La similitud más alta entre bosques se registró en otoño $(\mathrm{MH}=0.598)$ y la más baja en verano $(\mathrm{MH}=0.268)$.

El análisis de agrupamiento de acuerdo con la similitud (MH) de ambas localidades y estaciones climáticas se representa en un fenograma (Fig. 2). Se reconocen dos grupos, uno correspondiente a Laguna Brava (LB) y el otro a El Perichón (EP). En esta última localidad se 
$\left.\begin{array}{r}0.05 \\ 0.04 \\ 0.03 \\ 0.02 \\ 0.01 \\ 0\end{array}\right]$

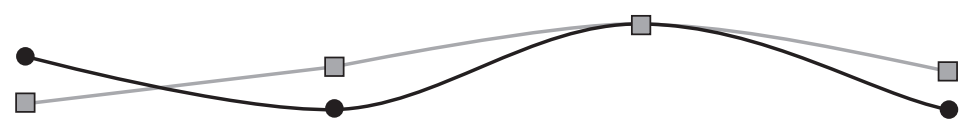

E $\left.\begin{array}{r}0.92 \\ 0.9 \\ 0.88 \\ 0.86 \\ 0.84 \\ 0.82 \\ 0.8\end{array}\right]$

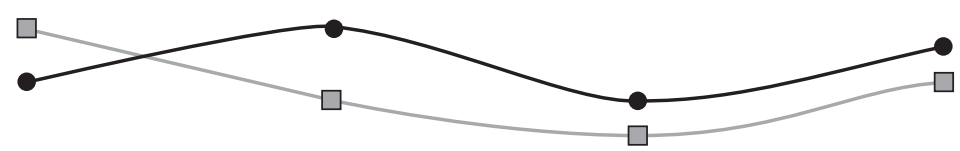

$\left.\mathbf{H}^{\prime} \begin{array}{r}3.9 \\ 3.8 \\ 3.6 \\ 3.5 \\ 3.4 \\ 3.3\end{array}\right]$

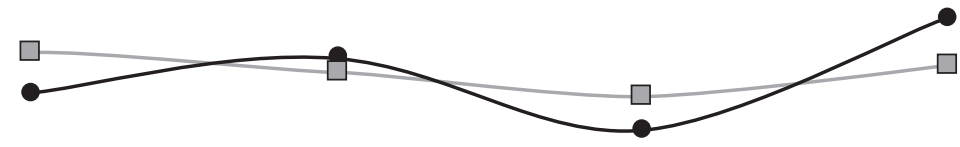

$\left.\mathbf{S} \begin{array}{l}75 \\ 70 \\ 65 \\ 60- \\ 55 \\ 50\end{array}\right]$
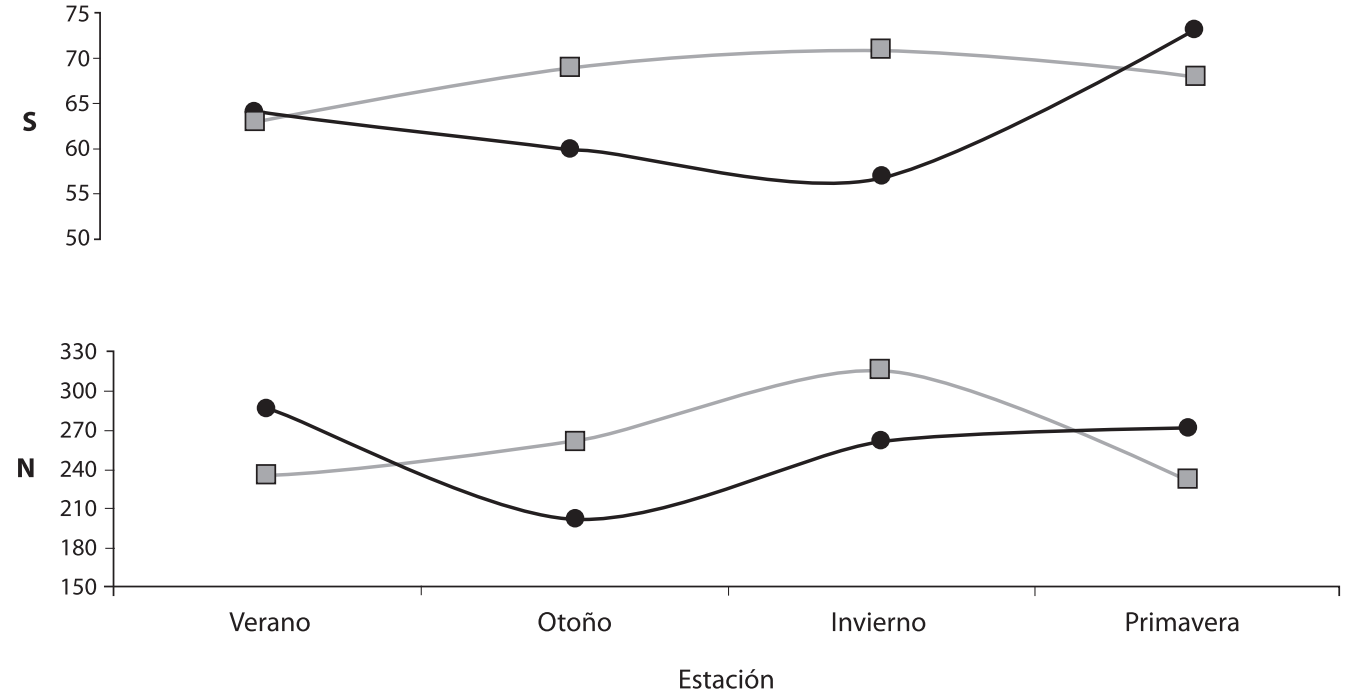

$\longrightarrow$ Lag. Brava

$\square-$ El Perichón

Fig. 1. Número de individuos $(\mathrm{N})$, riqueza específica $(\mathrm{S})$, diversidad (H', D) y equidad (E), según localidad y estación climática (Corrientes, Argentina) (2001-2002).

Fig. 1. Number of individual $(\mathrm{N})$, specific richness $(\mathrm{S})$, diversity $\left(\mathrm{H}^{\prime}, \mathrm{D}\right)$ and equity $(\mathrm{E})$, according to locality and climatic station (Corrientes, Argentina)(2001-2002). 


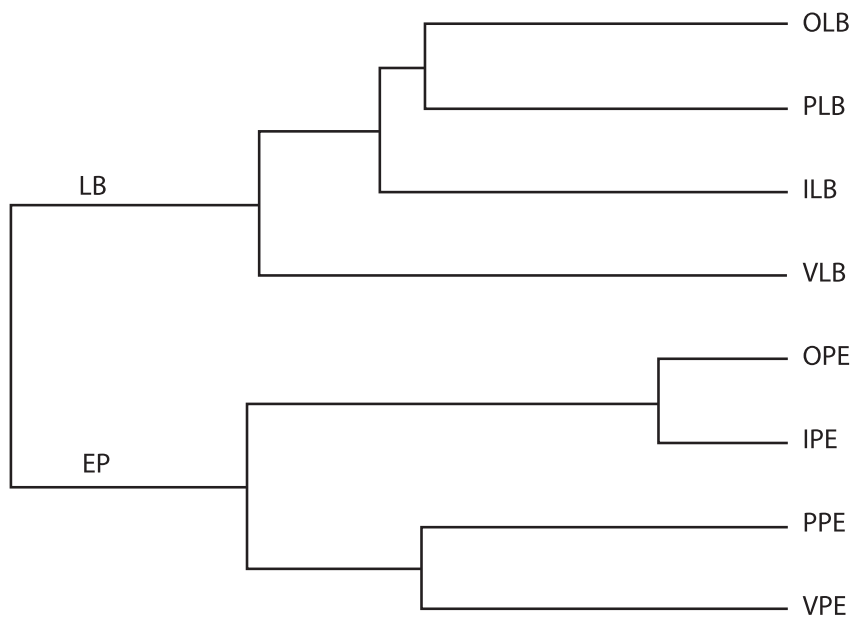

0.950

Fig. 2. Análisis de agrupamiento de arañas de acuerdo a la similitud $(\mathrm{MH})$ y estaciones climáticas en Laguna Brava (LB) y El Perichón (PE) (Corrientes, Argentina). OLB y OPE: otoño, PLB y PPE: primavera, VLB y VPE: verano, ILB e IPE: invierno.

Fig. 2. Cluster analisis of spider similarity $(\mathrm{MH})$ and climatic stations in Laguna Brava (LB) and El Perichón (PE)(Corrientes, Argentina). OLB and OPE: autumn, SLB and SPE: spring, VLB and VPE: summer, ILB and IPE: winter.

unieron las estaciones otoño (OPE) con invierno (IPE) y primavera (PPE) con verano (VPE). En Laguna Brava no se observan estas afinidades y el verano (VLB) mostró baja similitud con las demás estaciones. El índice de Chao 1 indica que las especies halladas representan el $81 \%$ y el $82 \%$ de la riqueza estimada en Laguna Brava y El Perichón, respectivamente.

\section{DISCUSIÓN}

Desde el punto de vista taxonómico, las familias de arañas encontradas en este trabajo representan aproximadamente el $53 \%$ del total de familias identificadas hasta el presente para Argentina (Platnick 2006). El total de familias recolectadas es significativo si lo comparamos con los resultados obtenidos por Corronca y Abdala (1994). Estos autores, mediante un relevamiento de la araneofauna en la Reserva El Bagual, Formosa, encontraron 23 familias al analizar diferentes hábitats con muestreos intensivos. No obstante, debido al escaso estudio de la araneofauna en bosques naturales en Argentina, no sería conveniente sacar conclusiones sin un marco comparativo que permita hacer un análisis más exhaustivo.

En coincidencia con lo observado por Costa et al. (1991), en la diversidad de familias recolectadas en ambos bosques, probablemente hayan incidido factores como humedad relativa, luz u otros vinculados con la cobertura vegetal; esta relación general quizás refleje la mayor cantidad y diversidad de nichos disponibles para las arañas en los hábitats más complejos y con menor frecuencia de disturbios. Esto es coincidente con lo que sostiene Desender et al. (1989).

Al comparar los resultados con otros trabajos realizados en otros países, en ambientes con características parecidas, se observó que las familias halladas en ambos bosques, son similares a las registradas por Silva (1996) en un bosque tropical del Perú, empleando técnicas de fumigación, agitación del follaje y barridos con red; esta autora obtiene una diversidad alta para las familias Araneidae, Theridiidae y Salticidae. En otro trabajo, Silva y Coddington (1996), realizado en Pakitza, Perú, obtienen resultados semejantes.

Coincidiendo con Duffey (1962) y Breymeyer (1966) que sostienen que los adultos no superan el $48 \%$ de las poblaciones naturales de Araneomorphae, en esta investigación la población adulta alcanzó el $26 \%$ sumando las dos áreas de estudio. 
Los artrópodos se dispersan por medio de troncos $\mathrm{u}$ otra vegetación flotante sobre ambientes lóticos (Poi de Neiff 2003). El río Paraná atraviesa bosques de Brasil y Misiones (Argentina) transportando diversos elementos que sirven de medio de dispersión a la artropofauna. Este hecho podría justificar la presencia de familias, típicas de bosques más húmedos, como Theridiosomatidae, Deinopidae y especies del género Amazonepeira (Araneidae) en el bosque de El Perichón, ubicado en cercanía de este río.

La complejidad de las estructuras del bosque parece ejercer un efecto directo sobre el emplazamiento de las telas, y en general, el aumento en la disponibilidad de sustratos donde fijarlas probablemente cause un aumento en la densidad de las arañas (Rypstra 1985, Samu y Szinetár 2002). Esta postura parece coincidir con la gran densidad de arañas que pertenecen a los gremios constructoras de telas orbiculares y constructoras de telas sabanas, halladas en esta investigación.

El mayor número de individuos registrado en invierno en El Perichón y el número más o menos constantes de arañas halladas en las otras estaciones climáticas, indicaría que los bosques funcionan como hábitats de microclimas estables y sitio de preferencia para el establecimiento de la fauna. Además, la permanente cobertura del suelo, la cual provee refugio y disponibilidad de presas, facilitaría la aireación y la regulación de la temperatura ambiente, haciendo a estas áreas más propicias para el desarrollo de las arañas (Duffey 1978, Gravesen y Toft 1987, Desender et al. 1989). Asimismo, la abundancia de arañas en el verano del bosque Laguna Brava puede asociarse a la disponibilidad de presas en esta época del año (Uetz 1976, Riechert y Luczak 1982).

Los valores más altos registrados con el índice de Simpson (D) en invierno en ambas localidades están dados por un incremento en el número de individuos de Tmarus sp.1 y Aysha sp. en Laguna Brava y Aysha sp. y msp no2 (Theridiidae) en El Perichón.
El elevado número de taxones representados por un solo individuo y dos individuos, coincide con la hipótesis de que la artropofauna tropical se caracteriza por presentar un alto número de especies, las cuales en su mayoría exhiben bajas densidades poblacionales o son raras (Flórez 1998). Resultados similares en bosques neotropicales han sido obtenidos por Silva (1996) y Silva y Coddington (1996).

Según Ruzicka (1987) Lycosidae y Linyphiidae no superan juntas el $45 \%$ de los individuos en áreas protegidas, alcanzando el $85 \%$ en áreas deterioradas. Si bien no se utilizaron trampas de caída que sobrestiman los muestreos de licósidos (Dinter 1995), dichas familias alcanzan el $14 \%$ y $8 \%$ en Laguna Brava y El Perichón respectivamente. Los porcentajes presentados indicarían un bajo grado de deterioro en los bosques estudiados, en relación con los valores obtenidos (54.41\%) por Pérez-Miles et al. (1999) en ambientes urbanizados.

El análisis de agrupamiento demuestra una disposición de las estaciones climáticas en dos grupos (correspondientes a ambas localidades) lo que permite reflejar la araneofauna propia de cada bosque.

Si bien no responde a lo observado en Laguna Brava, la similitud entre otoño e invierno (OPE-IPE) y primavera y verano (PPEVPE) en El Perichón podría explicarse por la estacionalidad seca-lluviosa propia de esta región del país.

Teniendo en cuenta la abundancia y la riqueza de arañas halladas en las áreas estudiadas, son necesarias investigaciones futuras en bosques naturales que permitan tener un panorama más amplio y hacer comparaciones objetivas. Además, estudios realizados en diferentes ecosistemas naturales de Europa han demostrado que las arañas alcanzan alta densidad cuando los disturbios causados por la actividad humana son mínimos (Kajak 1971, Lohmeyer y Pretscher 1979). Por ello es fundamental la conservación de estos ambientes naturales, actualmente desprotegidos. 


\section{RESUMEN}

El importante avance de la degradación de bosques en la Provincia Biogeográfica del Chaco, Argentina, conlleva una pérdida importante de su poco conocida biodiversidad. Se realizó un estudio de la fauna de arañas en dos bosques de la provincia de Corrientes, Argentina, correspondientes al Distrito Oriental Húmedo del Chaco, en las localidades de Laguna Brava y El Perichón. Se realizaron muestreos estacionales entre los años 2001 y 2002. Las arañas recolectadas (2 067 individuos de 33 familias y 226 especies/ morfoespecies) fueron obtenidas por golpeteo del estrato arbustivo y tamizado de hojarasca. Las familias Araneidae, Anyphaenidae, Salticidae y Theridiidae fueron las más abundantes en los dos bosques. El gremio de arañas "constructoras de telas orbiculares" presentó el mayor número de individuos $(\mathrm{n}=382)$ y el de "cazadoras al acecho" la mayor riqueza $(\mathrm{S}=56)$. En Laguna Brava se observó mayor abundancia en el verano $(n=287)$ y primavera $(n=273)$ y en el Perichón en invierno $(n=315)$. La riqueza específica y el valor de los índices de diversidad, fueron mayores en Laguna Brava $\left(\mathrm{S}=134, \mathrm{H}^{\prime}=4.23, \mathrm{E}=0.86, \mathrm{D}=0.023\right)$ que en El Perichón $\left(\mathrm{S}=127, \mathrm{H}^{\prime}=4.08, \mathrm{E}=0.84, \mathrm{D}=0.029\right)$. La similitud entre ambos bosques fue $\mathrm{MH}=0.611$.

Palabras clave: Araneae, biodiversidad, bosques húmedos, Corrientes, Argentina.

\section{REFERENCIAS}

Barnes, B.V., D.R. Zak, S.R. Denton \& S.H. Spurr. 1998. Forest ecology. Wiley, Nueva York, EEUU.

Breymeyer, A. 1966. Relations between wandering spiders y other epigeic predatory Arthropoda. Ekol. Pol. 14: $27-7$.

Bruniard, E. 1981. El clima de las planicies del norte argentino. Editorial Universitaria de la Universidad Nacional del Nordeste, Resistencia, Argentina.

Cabrera, A.L. \& A. Willink. 1980. Biogeografía de América Latina. OEA, Serie de Biología, Monografía 13, Washington D.C., EEUU.

Clausen, I.H.S. 1986. The use of spiders (Araneae) as ecological indicators. Bull. British Arachnol. Soc. 7: 83-86.

Coddington, J.A. \& H.W. Levi. 1991. Systematics y evolution of spiders (Araneae). Annu. Rev. Ecol. Syst. 22: 565-592.

Colwell, R. \& J.A. Coddington. 1994. Estimating terrestrial biodiversity through extrapolation. Phil. Trans. R. Soc. London 345: 102-118.
Corronca, J.A. \& C.S. Abdala. 1994. La fauna araneológica de la Reserva Ecológica "El Bagual", Formosa, Argentina. Aracnología Supl. 9: 1-6.

Costa, F.G., F. Pérez-Miles, E. Gudynas, L. Prandi \& R.M. Capocasale. 1991. Ecología de los arácnidos criptozoicos, excepto ácaros, de Sierra de las Animas (Uruguay). Ordenes y familias. Aracnol. 13/15: 1-14.

Desender, K., M. Alderweireldt \& M. Pollet. 1989. Field edges y their importance for polyphagos predatory arthropods. Med. Fac. Landbouww. Rijksuniv. Gent. 54: 823-833.

Dinter, A. 1995. Estimation of epigeic spider population densities using an intensive D-vac sampling technique y comparison with pitfall trap catches in winter wheat. Arthropod natural enemies in arable land I. Acta Jutlandica 70: 23-32.

Dippenaar-Schoeman, A.S., A.M. Van Den Berg \& A. Van Den Berg. 1989. Species composition y relative seasonal abundance of spiders from the field $y$ tree layers of the Roodeplaat Dam Nature Reserve. Koedoe 32: 25-38.

Duffey, E. 1962. A population study of spiders in limestone grassland, the fiel-layer fauna. Oikos 13: 15-34.

Duffey, E. 1978. Ecological Strategies in spiders including some characteristics of species in pioneer y nature habitats. Symp. Zool. Soc London 42: 109-123.

Enders, F. 1976. Clutch Size Related to Hunting Manner of Spider Species. Ann. Entomol. Soc. Am. 69: 991998.

Flórez, D.E. 1998. Estructura de comunidades de arañas (Araneae) en el departamento Del Valle, suroccidente de Colombia. Caldasia 20: 173-192.

Gravesen, E. \& S. Toft. 1987. Grassfields as reservoirs for polyphagous predators (Arthropoda) of aphids (Homoptera, Aphididae) J. Appl. Entomol. 104: 461473.

Hatley, C.L. \& J.A. Macmahon. 1980. Spider community organization: seasonal variation y the role of vegetation archtecture. Environ. Entomol. 9: 632-639.

Höfer, H. 1990. The spider community (Araneae) of a central Amazonian blackwater inundation forest (Igapó). Acta Zool. Fennica 190: 173-179.

Kajak, A. 1971. Productivity investigation of two types of meadown in the Vistula Valley. IX

Production y consumption of field layer spiders. Ekol. Pol. 19: 197-211. 
Lohmeyer, W. \& P. Pretscher. 1979. Über das Zustandekommen Wildstauden-Quecken-Fluren auf Brachland in Boon und ihre Bedeutunng als Lebensraum für die Wespenpinne. Natur Landschaft 54: 253-259.

Maelfait, J.P., R. Jocque, L. Baert \& K. Desender. 1990. Heathland management y spiders. Acta Zool. Fennica 190: 261-166.

Marc, P. \& A. Canard. 1997. Maintaining spider biodiversity in agroecosystems as a tool in pest control. Agriculture. Ecosyst. Environ. 62: 229-235.

Meffe, G.K. \& C.R. Carroll. 1994. Principles of Conservation Biology. Sinauer Associates, Sunderland, Massachusetts, EEUU.

Morrone, J.J. 2001. Biogeografía de América Latina y del Caribe. Manuales y Tesis de la SEA, CYTED, ORCYT- UNESCO, Zaragoza, España.

Nyffeler, M.W., W.L. Sterling \& D. Dean. 1994. How spiders make a living. Environ. Entomol. 23: 13571367.

Pérez-Miles, F., M. Simó, C. Toscano-Gadea \& G. Useta. 1999. La comunidad de Araneae criptozóicas del Cerro de Montevideo, Uruguay: Un ambiente rodeado por urbanización. PHYSIS C 57: 73-78.

Pinkus-Rendón, M.A., J.L. León-Cortés \& G. IbarraNúñez. 2006. Spider diversity in a tropical habitat gradient in Chiapas, mexico. Diversity Distrib. 12: 61:69.

Poi de Neiff, A. 2003. Macroinvertebrates living on Eichhornia azurea Kunth in the Paraguay River. Acta Limnol. Bras. 15: 55-63.

Riechert, S.E. \& J. Luczak. 1982. Spider foraging: behavioral responses to prey, p. 353-385. In P.N. Witt \& J. S. Rovner (eds.). Spider comunication. Mechanisms y ecological significance. Princeton, Nueva Jersey, EEUU.

Ruzicka, V. 1987. Biodiagnostic evaluation of epigeic spider communities. Ekológia (CSSR) 6: 345-357.

Rypstra, A. 1985. Agg of Nephila clavipes (L.) (Araneae: Araneidae) in relation to prey availability. J. Arachnol. 13: 71-78.
Samu, F. \& C. Szinetar. 2002. On the nature of agrobiont spiders. J. Arahnol. 30: 389-402.

Scheidler, M. 1990. Influence of habitat structure y vegetation architecture on spiders. Zool. Anz. 5/6: 333 340 .

Silva, D. 1996. Species composition y community structure of Peruvian rainforest spiders: a case study from a seasonally inundated forest along the Samiria river. Rev. Suis. Zool. vol hors serie 2: 597-610.

Silva, D. \& J.A. Coddington. 1996. Spiders of Pakitza (Madre de Dios, Peru): species richness y notes in community structure, p 241-299. In D.E. Wilson \& A. Sandoval (eds.). The biodiversity of Pakitza y its environs. Smithsonian Institution, Washington, EEUU.

Tsai, Z.I., P.S. Huang \& I.M. Tso. 2006. Habitat management by aboriginals promotes high spider diversity on an Asian tropical island. Ecography 29: 84-94.

Turnbull, A.L. 1973. Ecology of the true spiders (Araneomorphae). Annu. Rev. Entomol. 18: 305-348.

Uetz, G.W. 1976 Gradient analysis of spider communities in a streamside forest. Oecologia (Berl.) 22: 373-385.

Uetz, G.W., J. Halaj \& A.B. Cady. 1999. Pitfal traping in ecological studies of wandering spider. J. Arachnol. 27: $270-280$.

Weeks, R.D. Jr. \& T.O. Holtzer. 2000. Habitat y Season in Structuring Ground-Dwelling Spider (Araneae) Communities in a Shortgrass Steppe Ecosyst. Environ. Entomol. 29: 1164-1172.

Willett, T.R. 2001. Spiders y other arthropods as indicators in old-growth versus logged redwood stands. Restoration Ecology 9: 410-420.

Wise, D. 2002. Efectos directos e indirectos de las arañas en la red trófica del mantillo del bosque. V Congr. Argent. de Entomol., Buenos Aires, Argentina.

\section{REFERENCIA DE INTERNET}

Platnick, N.I. 2006. The world spider catalog. Versión 6.5. Am. Mus. Nat. Hist. (Consultado: http://research. amnh.org/entomology/spiders/catalog/index.html) 
